\title{
Evidence for Phosphate Starvation of Rhizobia without Terminal Differentiation in Legume Nodules
}

\author{
Yue Hu, Jian Jiao, Li Xue Liu, Yan Wei Sun, Wen Feng Chen, Xin Hua Sui, Wen Xin Chen, and \\ Chang Fu Tian ${ }^{+}$
}

State Key Laboratory of Agrobiotechnology, MOA Key Laboratory of Soil Microbiology, Rhizobium Research Center, and College of Biological Sciences, China Agricultural University, 100193, Beijing, China

Accepted 13 April 2018.

\begin{abstract}
Phosphate homeostasis is tightly modulated in all organisms, including bacteria, which harbor both high- and low-affinity transporters acting under conditions of fluctuating phosphate levels. It was thought that nitrogen-fixing rhizobia, named bacteroids, inhabiting root nodules of legumes are not phosphate limited. Here, we show that the high-affinity phosphate transporter PstSCAB, rather than the low-affinity phosphate transporter Pit, is essential for effective nitrogen fixation of Sinorhizobium fredii in soybean nodules. Symbiotic and growth defects of the pst mutant can be effectively restored by knocking out PhoB, the transcriptional repressor of pit. The pst homologs of representative rhizobia were actively transcribed in bacteroids without terminal differentiation in nodules of diverse legumes (soybean, pigeonpea, cowpea, common bean, and Sophora flavescens) but exhibited a basal expression level in terminally differentiated bacteroids (alfalfa, pea, and peanut). Rhizobium leguminosarum bv. viciae Rlv3841 undergoes characteristic nonterminal and terminal differentiations in nodules of $S$. flavescens and pea, respectively. The pst mutant of RIv3841 showed impaired adaptation to the nodule environment of S. flavescens but was indistinguishable from the wildtype strain in pea nodules. Taken together, root nodule rhizobia can be either phosphate limited or nonlimited regarding the rhizobial differentiation fate, which is a host-dependent feature.
\end{abstract}

Inorganic phosphate $(\mathrm{Pi})$ is essential for maintaining cellular structure and energy currency in all organisms (Qi et al. 2016). Pi sensing and signaling has been intensively investigated in the model unicellular microorganism Escherichia coli. Under high Pi conditions, Pi binding to the high-affinity Pi transporter PstSCAB can inhibit the histidine kinase PhoR, leading to the inactivation of transcription factor $\mathrm{PhoB}$ and subsequent repression of pstSCAB transcription and derepression of the low-affinity Pi transporter Pit (McCleary 2017; Taylor et al. 2014). Under Pi-limited conditions, the repression on PhoR

${ }^{\dagger}$ Corresponding author: Chang Fu Tian; E-mail: cftian@cau.edu.cn

Funding: This work was supported by the National Natural Science Foundation of China (31570069) and the National Basic Research Program of China (973 program 2015CB158301).

*The $\boldsymbol{e}$-Xtra logo stands for "electronic extra" and indicates that two supplementary tables are published online.

() 2018 The American Phytopathological Society autophosphorylation is released, allowing activation of $\mathrm{PhoB}$ and PhoB-mediated modulation of Pho regulon (SantosBeneit 2015). The extensive studies of diverse bacterial pathogens encountering either Pi-limiting or Pi-rich environments in eukaryote hosts have further revealed an adaptive role of bacterial $\mathrm{Pi}$ sensing or signaling in stress response and virulence (Lamarche et al. 2008; Taylor et al. 2014). However, our understanding of $\mathrm{Pi}$ homeostasis in bacteria symbiotically associated with eukaryotes is limited.

Rhizobia are characterized by their ability of forming nitrogen-fixing nodules on legume roots and play important roles in sustainable agriculture and global ecology. Within nodule cells, differentiated rhizobia named bacteroids can fix atmospheric nitrogen to support plant growth, while the host provides carbon sources and essential nutrients to bacteroids to support the energy-consuming process of nitrogen fixation (Udvardi and Poole 2013).The morphotype and fate of bacteroids are governed by the varying number and type of symbiotic nodule-specific cysteine-rich (NCR) peptides in different legumes (Montiel et al. 2017). Within Medicago root nodules, bacteroids of the model rhizobium Sinorhizobium meliloti undergo an irreversible (terminal) differentiation characterized by enlarged cell size and impaired reproductive ability (Van de Velde et al. 2010). The terminal differentiation of bacteroids can also be found in other legumes such as species of Pisum, Trifolium, Aeschynomene, and Arachis, but not in Lotus, Glycine, Phaseolus, Vigna, Cajanus, Leucaena, Sophora, and so on (Crespo-Rivas et al. 2016; Li et al. 2013; Montiel et al. 2016; Oono et al. 2010).

Despite a great diversity of rhizobium-legume symbioses (Alunni and Gourion 2016; Haag et al. 2013; Remigi et al. 2016) and the fact that approximately $70 \%$ of global cultivated land is deficient in Pi (López-Arredondo et al. 2014), our knowledge of rhizobial Pi homeostasis is restricted in S. meliloti, a natural microsymbiont of Medicago sativa The low-affinity orfA-pit-encoded Pi uptake system Pit rather than the high-affinity transporters PstSCAB and PhoCDET is expressed in nodules and is sufficient for symbiosis of $S$. meliloti bacteroids (Bardin et al. 1996; diCenzo et al. 2017; Yuan et al. 2006), and it has been consequently proposed that root nodule bacteria are not Pi limited (Udvardi and Poole 2013). However, a recent transcriptomic analysis of $S$. fredii bacteroids in Glycine max (soybean) nodules suggested active expression of pstSCAB and phoCDET but very little expression of orfA-pit genes (Jiao et al. 2016), and the pstSCAB operon is essential for the nitrogen fixation process (Jiao et al. 2016). This highlights that bacteroids may encounter contrasting nodule environments of different legume hosts. 
In this study, we aimed to find a clue about the Pi homeostasis of bacteroids associated with different legume hosts. First, various single and combined deletion mutants of highand low-affinity Pi transporters and PhoB were constructed in $S$. fredii and tested for their symbiotic performance on G. max. Together with in vivo measurement of the expression of different transporters in nodules induced by the wild-type $S$. fredii and the $p h o B$ mutant, it was demonstrated that $S$. fredii bacteroids in G. max nodules were Pi limited. The expression level of rhizobial high-affinity Pi transporter Pst in nodules of Cajanus cajan (pigeonpea), Phaseolus vulgaris (common bean), Vigna unguiculata (cowpea), Pisum sativum (pea), Sophora flavescens, Arachis hypogaea (peanut), and Medicago sativa (alfalfa) was further determined to test its potential correlation with the types of nodule development (with either persistent or transient meristems) or the differentiation fate of bacteroids (with or without terminal differentiation). By using Rhizobium leguminosarum bv. viciae 3841 (Rlv3841) inducing indeterminate nodules on both $P$. sativum and $S$. flavescens, it was further uncovered that the differentiation fate of bacteroids is related to the observed differential expression of Pst in bacteroids. The significance of our findings was further discussed in the context of coevolution of two symbiotic partners.

\section{RESULTS}

\section{Symbiotic defects of the pst mutant}

of $S$. fredii can be recovered by the deletion of phoB.

The $S$. fredii CCBAU45436 (abbreviated as SF45436) genome harbors genes encoding the conserved high-affinity $\mathrm{Pi}$ transporters PstSCAB and PhoCDET and an orfA-pit encoded low-affinity Pit transport system, which showed 91.82 to 97.42 , 79.45 to 95.35 , and 91.59 to $93.41 \%$ identity, respectively, to their homologous proteins of Sinorhizobium meliloti 2011 (Fig. 1). We had previously demonstrated an impaired symbiotic performance of the pst mutant of SF45436 on G. max but a mutant lacking the key regulator $\mathrm{PhoB}$ was still an efficient microsymbiont (Jiao et al. 2016). To determine whether PhoCDET or Pit also contribute to the symbiosis of SF45436 in G. max nodules, phoC and pit were individually deleted in the wild-type SF45436 and the pst mutant. The phoC and pit mutants were not significantly different from SF45436 regarding shoot dry weight and nodule fresh weight of inoculated $G$. max plants at 30 days postinoculation (dpi) ( $P$ values $>0.05, t$ test) (Fig. $2 \mathrm{~A}$ and $\mathrm{B}$ ). Like SF45436 and the $p h o B$ mutant, multiple bacteroids within each symbiosome can be found in nodule cells infected by the phoC and pit mutants (Fig. 2C). The shoot dry weight and nodule fresh weight of plants inoculated with double mutants pst-pit and pst-phoC were significantly lower than SF45436 ( $P$ values $<0.05$, $t$ test) (Fig. 2A and B). In contrast to the pst mutant, many bacteroids of irregular shape were observed for the pst-pit and pstphoC mutants, and the number of bacteroids per symbiosome was increased in nodules infected by the pst-phoC mutant (mean \pm standard error, $3.2 \pm 0.2$ compared with $1.7 \pm 0.1$ for the pst mutant; $P$ value $<0.001, t$ test with 100 scored symbiosomes) (Fig. 2C), indicating a contribution of Pit and PhoCDET to rhizobial adaptation to nodule environments.

Because PhoB acts as a positive regulator for pstSCAB and phoCDET but represses the transcription of pit (Bardin and Finan 1998; Krol and Becker 2004; Yuan et al. 2006), phoB-pst, phoB-phoC, and phoB-phoC-pst mutants were constructed to test whether symbiotic defects associated with pst could be recovered. It turned out that these mutants were as effective as SF45436 when inoculated on the test $G$. $\max$ cultivar in terms of shoot dry weight and nodule fresh weight ( $P$ values $>0.05$, $t$ test) (Fig. 2A and B). The morphology or the number of bacteroids per symbiosome were partially recovered in the phoB- pst and phoB-phoC-pst mutants compared with pst and pst-phoC (Fig. 2C). To further verify the PhoB-dependent regulation of pstSCAB, phoCDET, and pit in nodules, an lacZ translational reporter lacking its start codon was fused after the promoter-start codon region of $p s t S$, phoC, or pit, and plasmids carrying these $l a c Z$ reporters were individually conjugated into SF45436 and the $p h o B$ mutant. Within G. max nodules collected at $30 \mathrm{dpi}$, pstS::lacZ and phoC::lacZ were highly expressed in SF45436 while significantly downregulated in the $p h o B$ mutant (Fig. 3). However, pit::lacZ was much more highly expressed than pst::lacZ and phoC::lacZ in nodules infected by the phoB mutant (Fig. 3).

\section{Deletion of phoB can fully recover growth defects of the pst mutant under excess but not limiting Pi conditions.}

The mutants pst, pst-pit, and pst-phoC exhibiting an impaired symbiotic performance on $G$. max were further compared with the wild-type SF45436 regarding the growth rate in minimum medium supplied with a limiting $(0.1 \mathrm{mM})$ (Fig. 4A) or excess (2 mM) (Fig. 4B) level of Pi. The pst-phoC double mutant lacking high-affinity $\mathrm{Pi}$ transporters showed the most serious delay of growth under both conditions. The pst and pst-pit mutants were indistinguishable from each other and grew more slowly than SF45436 but faster than the pst-phoC mutant under two conditions. The growth defect of the pst mutant under the excess Pi condition can be fully recovered by deleting phoB (Fig. 4B). Under the limiting Pi condition, the growth rate of the phoB-pst mutant was similar to SF45436 till the late exponential phase but slowed down at later stages (Fig. 4A), implying that the upregulated low-affinity Pi transporter Pit could only partially recover the growth defect of the pst mutant under the limiting $\mathrm{Pi}$ condition.

\section{Relative expression level of pst correlates with differentiation fates of bacteroids but not nodule development.}

The contrasting findings on the role of high- and low-affinity Pi transporters obtained in S. meliloti-Medicago sativa (Yuan et al. 2006) and S. fredii-G. max symbioses reminded us of their differences in nodule development and morphotype of bacteroids. To investigate whether bacteroids can be Pi limited or not in a manner depending on the type of nodule development (indeterminate versus determinate) or bacteroid differentiation (with or without terminal differentiation), the relative transcription levels of $p s t S$ homologs were measured in nodules of various representative symbioses using quantitative reversetranscription polymerase chain reaction (qRT-PCR) (Fig. 5).

The $p s t S$ of SF45436 was expressed at similar levels in determinate nodules of $G$. max and indeterminate nodules of C. cajan. The pstS1 of $R$. tropici CIAT $899^{\mathrm{T}}$ (Martínez-Romero et al. 1991) and pstS of Sinorhizobium sp. NGR234 (Pueppke and Broughton 1999) were actively transcribed in determinate nodules of Phaseolus vulgaris and $V$. unguiculata, respectively, while the pstS of S. meliloti 1021 (Galibert et al. 2001)

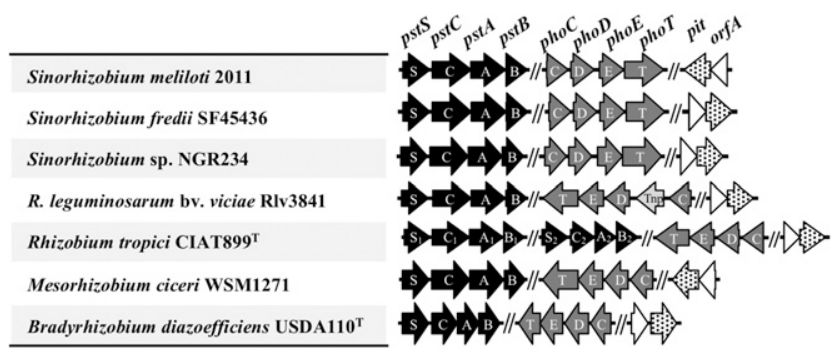

Fig. 1. Gene clusters encoding PstSCAB, PhoCDET, and Pit involved in phosphate transport in representative rhizobia. Tnp $=$ transposase. 
expressed at a relatively lower level in $M$. sativa indeterminate nodules (Fig. 5). Bradyrhizobium arachidis CCBAU051107 can induce determinate nodules on A. hypogaea and indeterminate nodules on Sophora flavescens (Jiao et al. 2015; Wang et al. 2013). The transcription level of pst $S$ gene of CCBAU051107 $7^{\mathrm{T}}$ was significantly higher in nodules of $S$. flavescens than in those of $A$. hypogaea $(P$ value $<0.001$, $t$ test). R. leguminosarum bv. viciae strains USDA2370 ${ }^{\mathrm{T}}$ and R3841 can form indeterminate nodules on Pisum sativum and S. flavescens (Jiao et al. 2015; Young et al. 2006), and their pst $S$ homologs were transcribed at a level significantly higher in $S$. flavescens than that in $P$. sativum ( $P$ values $<0.001, t$ test). The relative transcription levels of pst $S$ homologs were apparently unrelated to the type of nodule development. Instead, as indicated in Figure 5, they correlated with the fate of bacteroids (i.e., actively transcribed in nonterminally differentiated bacteroids while expressed at a lower level in terminally differentiated ones).

RIv3841 bacteroids are Pi limited in S. flavescens nodules but not in $P$. sativum nodules.

To get more insight into the host-dependent modulation of the expression of the high-affinity $\mathrm{Pi}$ transporter Pst, $S$. flavescens and $P$. sativum, forming indeterminate nodules with their common microsymbiont Rlv3841, were subject to further investigation. The lac Z reporter lacking its start codon was fused after the pst $S$ promoter-start codon region of Rlv3841, and the resulting pstS::lacZ translational reporter

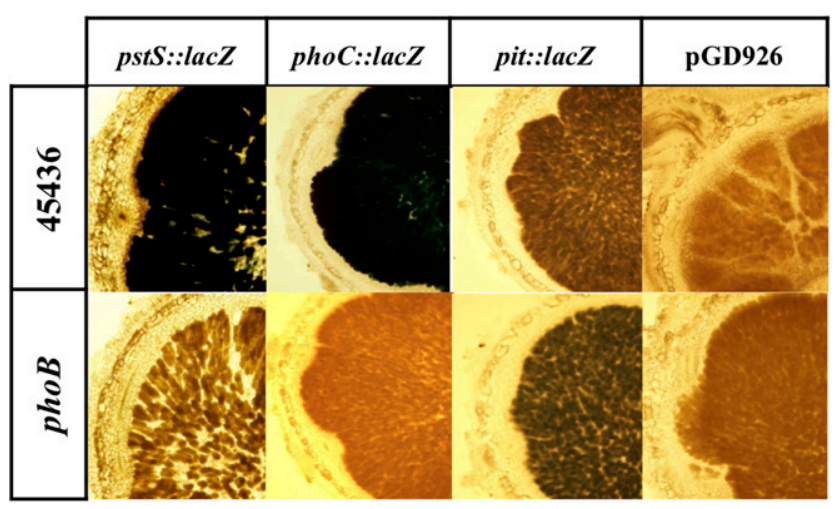

Fig. 3. Sections of soybean nodules stained for $\beta$-galactosidase activity. Nodules were collected 30 days postinoculation from plants inoculated with derivatives of the wild-type SF45436 and the phoB mutant harboring promoter lacZ fusions, including pstS::lacZ, phoC::lacZ, and pit::lacZ, and the pGD926 lacking a promoter for lacZ.
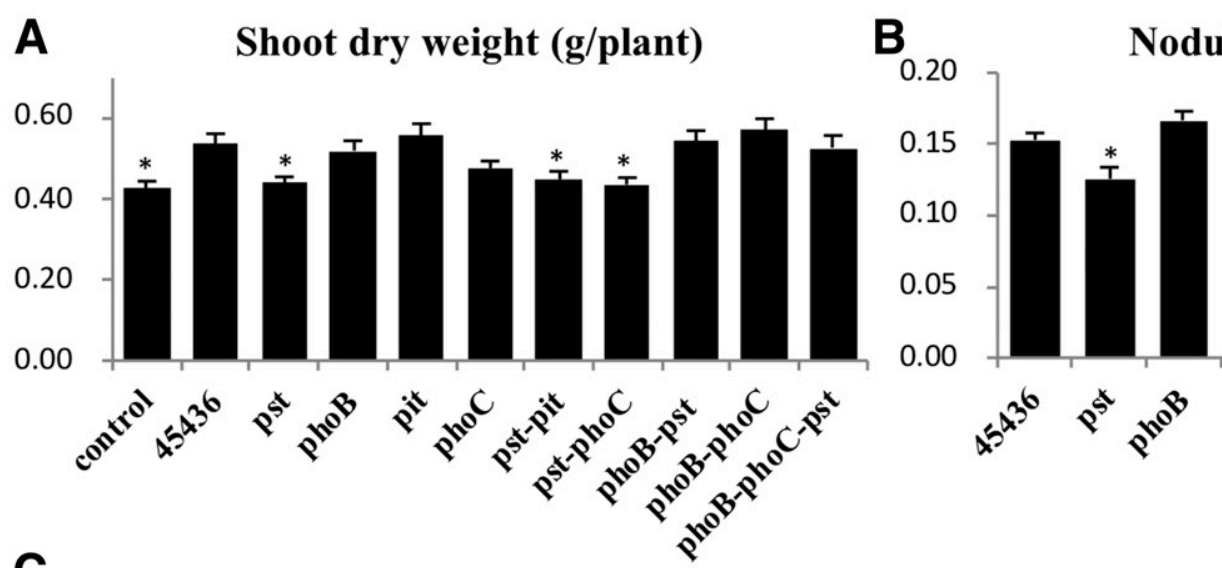

Nodule fresh weight (g/plant)
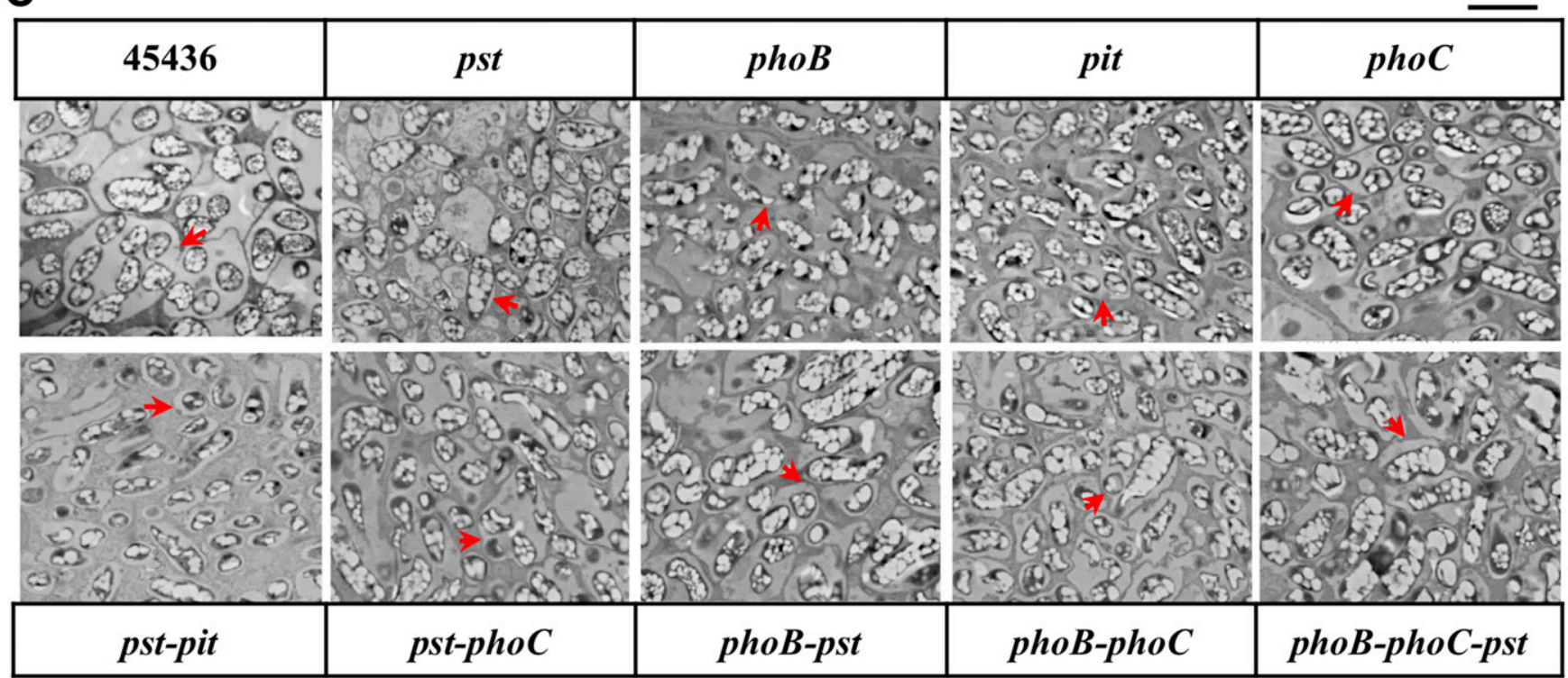

Fig. 2. Symbiotic performance of SF45436 and its derivatives on soybean. A, Shoot dry weight and B, nodule fresh weight per plant inoculated with SF45436 strains (30 plants from three independent experiments were scored). Significant difference between the mean of each treatment and the corresponding value of the wild-type SF45436 is indicated $(t$ test, $* P$ value $<0.05$ ). Error bars represent standard error of the means. C, Transmission electron microscopy pictures of $80-\mathrm{nm}$ thin section of soybean nodules ( 30 days postinoculation) formed by the wild-type SF45436 and its derivatives. Scale bar indicates $2 \mu \mathrm{m}$. Arrows point to symbiosome membrane. 
plasmid was conjugated into Rlv3841, allowing monitoring the expression level of $p s t S$ in vivo. At $40 \mathrm{dpi}$, the active expression of pstS was observed throughout the nodule section of $S$. flavescens (Fig. 6A). By contrast, $P$. sativum nodule cells generally exhibited a lower level of $p s t S$ expression, though those proximal infected cells corresponding to the senescent region of nodule had a relatively high $\beta$-galactosidase activity

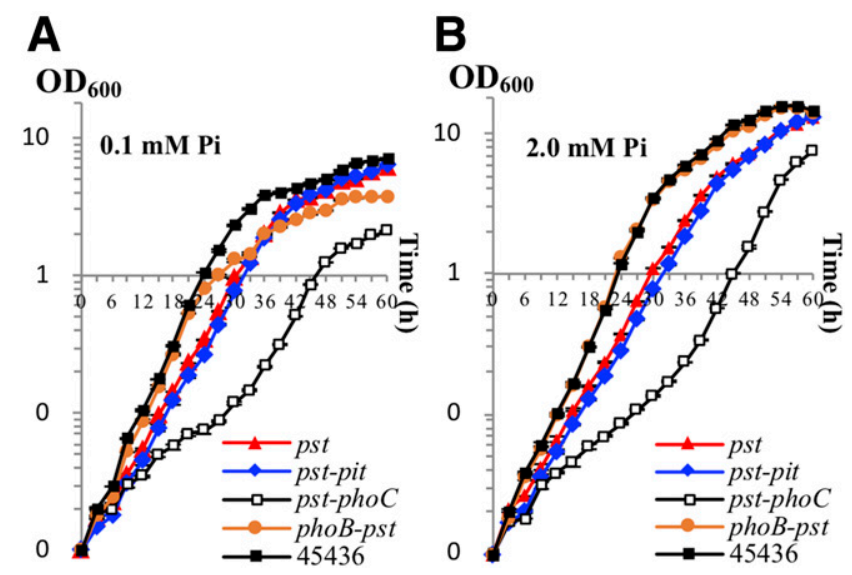

Fig. 4. Growth of SF45436 and its derivatives in minimal medium with limiting and excess inorganic phosphate $(\mathrm{Pi})$. Growth in minimal medium with A, 0.1 and B, $2 \mathrm{mM} \mathrm{Pi}$. $\mathrm{OD}_{600}=$ optical density at $600 \mathrm{~nm}$. Each symbol indicates the mean of three cultures, and error bars indicate standard errors. Similar results were obtained in two independent experiments.
(Fig. 6A). The qRT-PCR measurement of the transcription of pstS, phoC, and pit in free-living cultures of Rlv3841 under either limiting $(0.1 \mathrm{mM})$ or excess $(2 \mathrm{mM}) \mathrm{Pi}$ conditions demonstrated that $p s t S$ of Rlv3841 can be highly induced upon Pi starvation (Fig. 6B).

The legume nodule has been demonstrated to be a strong phosphorus sink compared with other organs under phosphorusdeficient conditions (Sulieman and Tran 2015). Here, the total phosphorus content of nodules, roots, or shoots was determined for $S$. flavescens and $P$. sativum plants grown under conditions supplied with 0,1 , or $2 \mathrm{mM}$ Pi (Fig. 6C). No detectable difference between two legume species in the phosphorus content of nodules could be observed under conditions with 0 or $1 \mathrm{mM}$ $\mathrm{Pi}$. When $2 \mathrm{mM}$ Pi was supplied, a notable increase of phosphorus content in nodule, root, and shoot was detected for two legume species compared with plants grown under conditions of lower Pi levels (Fig. 6C). P. sativum contained a higher concentration of phosphorus than $S$. flavescens in nodules and roots under the $2 \mathrm{mM}$ Pi condition $(P$ values $<0.001, t$ test) (Fig. 6C), indicating a higher Pi utilization efficiency by $P$. sativum. Moreover, a significant increase in shoot dry weight was observed under the $2 \mathrm{mM}$ Pi condition compared with the $0 \mathrm{mM}$ Pi condition for $P$. sativum $(P$ value $<0.001, t$ test) but not for $S$. flavescens $(P$ value $>0.05, t$ test $)$.

The size of Rlv3841 bacteroids associated with $P$. sativum but not those associated with $S$. flavescens was significantly larger than that of free-living cells $(P$ value $<0.001, t$ test $)$ (Fig. 6D). The number of CFU within $P$. sativum nodules was less than $5 \%$ of the number of CFU within S. flavescens nodules,

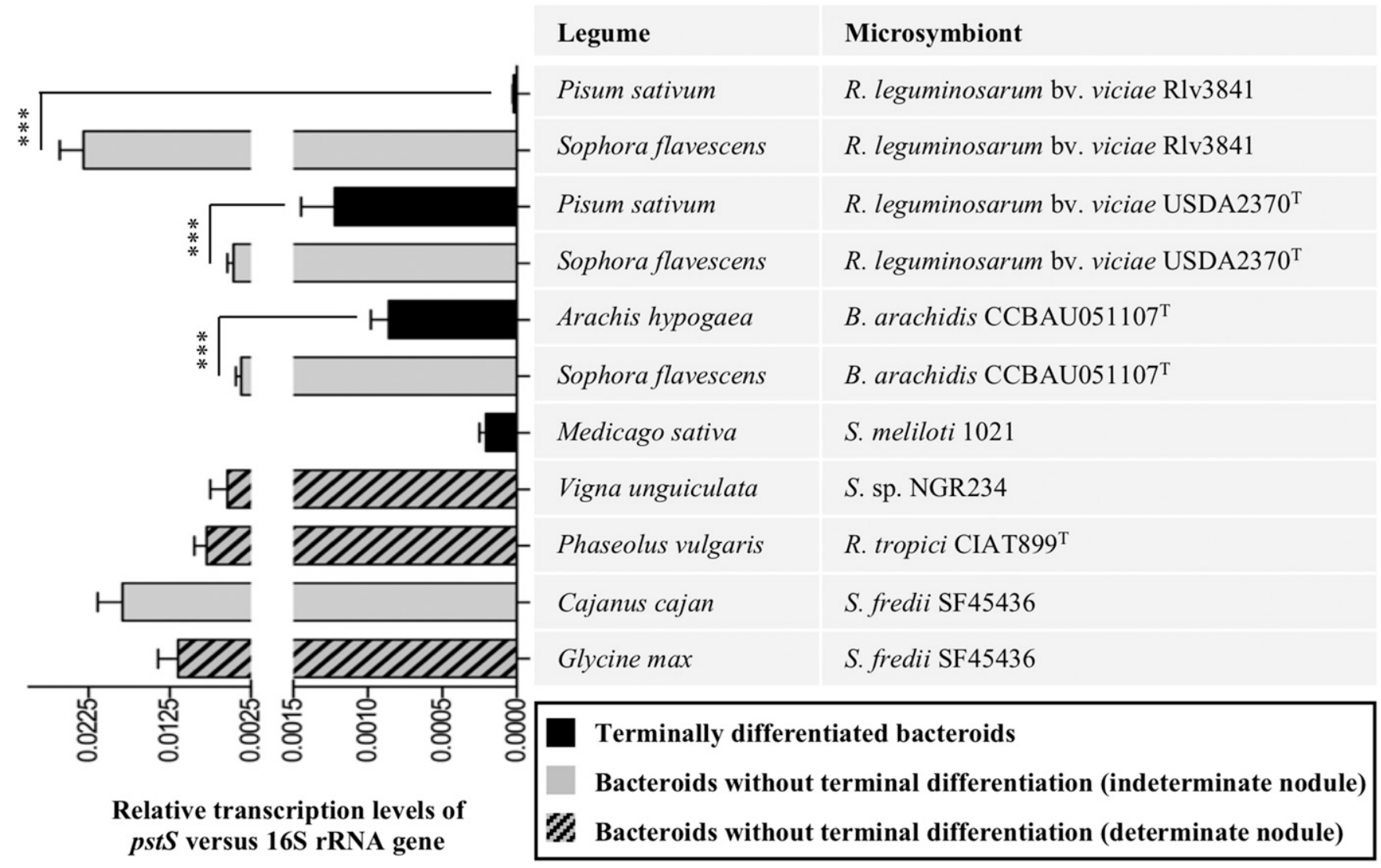

Fig. 5. Expression of $p s t S$ in bacteroids with and without terminal differentiation. Quantitative reverse-transcription polymerase chain reaction analysis of relative transcription levels of $p s t S$ versus $16 \mathrm{~S}$ ribosomal RNA (rRNA) gene. The $p s t S 2$ of $R$. tropici CIAT899 $9^{\mathrm{T}}$ was expressed at a basal level and only the result of pstS1 is shown herein. Means were based on nodule samples prepared from three independent inoculation experiments. Error bars represent standard error of mean, and significant difference is indicated $(t$ test, $* * *, P<0.001)$. The y-axis is presented in two segments of different major ticks intervals $($ left segment $=$ 0.01 and right segment $=0.0005$ ). 
either counted per nodule (Fig. 6E) or normalized by nodule fresh weight (mean \pm standard error, $0.32 \pm 0.09$ and $8.76 \pm$ 2.62 million CFU/mg in $P$. sativum and $S$. flavescens nodules, respectively) ( $P$ values $<0.01, t$ test on $\log _{10}$-transformed data).

To compare whether the pstS mutant of Rlv3841 would perform differentially within nodule environments of $S$. flavescens and $P$. sativum, ultrathin sections of 40-dpi nodules induced by the pstS mutant and the wild-type Rlv3841 were obtained (Fig. 6F). The pstS mutant was indistinguishable from the wild-type Rlv3841 in nodule cells of $P$. sativum, forming enlarged bacteroids. By contrast, the number of bacteroids harbored by each symbiosome membrane was significantly lower in $S$. flavescens nodules induced by the $p s t S$ mutant compared with that in nodules formed by the wild-type Rlv3841 ( $P$ value $<0.001, t$ test) (Fig. 6F and $\mathrm{G}$ ), indicating impaired adaptation of the pstS mutant. Under the test conditions, no significant differences between the pst $S$ mutant and the wild-type Rlv3841 could be detected regarding the shoot dry weight of inoculated $S$. flavescens and $P$. sativum plants ( $P$ values $>0.05, t$ test $)$.

\section{DISCUSSION}

It was proposed that root nodule bacteria are not Pi limited based on the findings obtained in Sinorhizobium melilotiMedicago sativa symbiosis (Udvardi and Poole 2013; Yuan et al. 2006). By contrast, this study uncovered that symbiotic bacteroids can be Pi limited in G. max nodules. However, the host-dependent expression profiles of the high-affinity $\mathrm{Pi}$ transporter Pst, sensing the extracellular Pi level, were independent of the type of nodule development (i.e., determinate or indeterminate). More generally, Pst was expressed lower in terminally differentiated bacteroids than in those without terminal differentiation. This was further highlighted by the expression pattern of Rlv3841 Pst across longitudinal nodule sections of $P$. sativum and Sophora flavescens, both of which form indeterminate nodules but show different Pi use efficiency. Moreover, the pstS mutant of Rlv3841 was impaired in adaptation to nodule environment of S. flavescens but not to that of $P$. sativum. These findings shed new light on our understanding of Pi homeostasis of bacteroids within legume nodules.

Inductions of strong phosphorus sink in nodules have been reported for different legumes, including $M$. sativa, M. truncatula, G. max, and Phaseolus vulgaris, under phosphorus-deficient conditions (Kouas et al. 2005; Sa and Israel 1991; Sulieman et al. 2013). This was further supported by this study of phosphorus content in different organs of Pisum sativum and S. flavescens plants (i.e., approximately twofold higher phosphorus concentration can be observed in nodules compared with roots and shoots under $0 \mathrm{mM}$ Pi conditions). Although there was no significant difference between $P$. sativum and $S$. flavescens in their total nodule phosphorus content under 0 and $1 \mathrm{mM}$ Pi conditions, the expression patterns of Rlv3841 pstS::lacZ in nodules
(A)

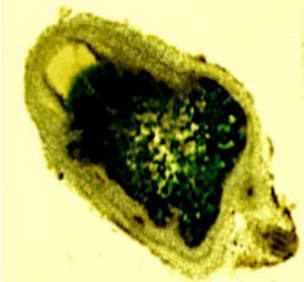

S. flavescens
(B)

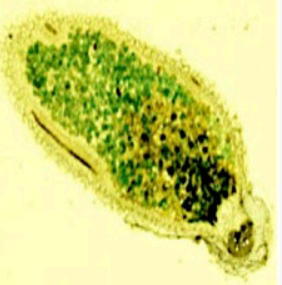

P. sativum

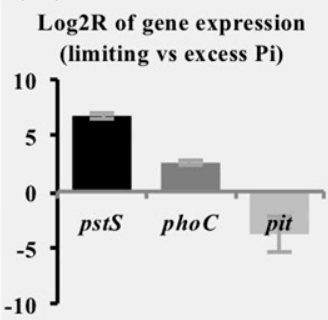

(D)

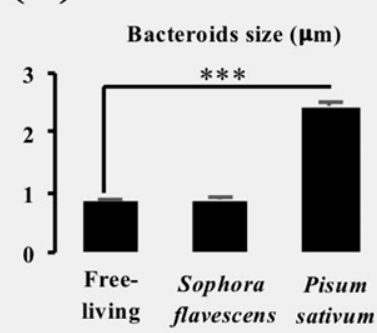

(E)

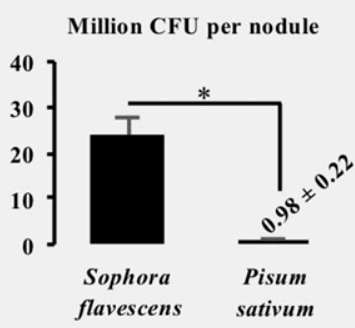

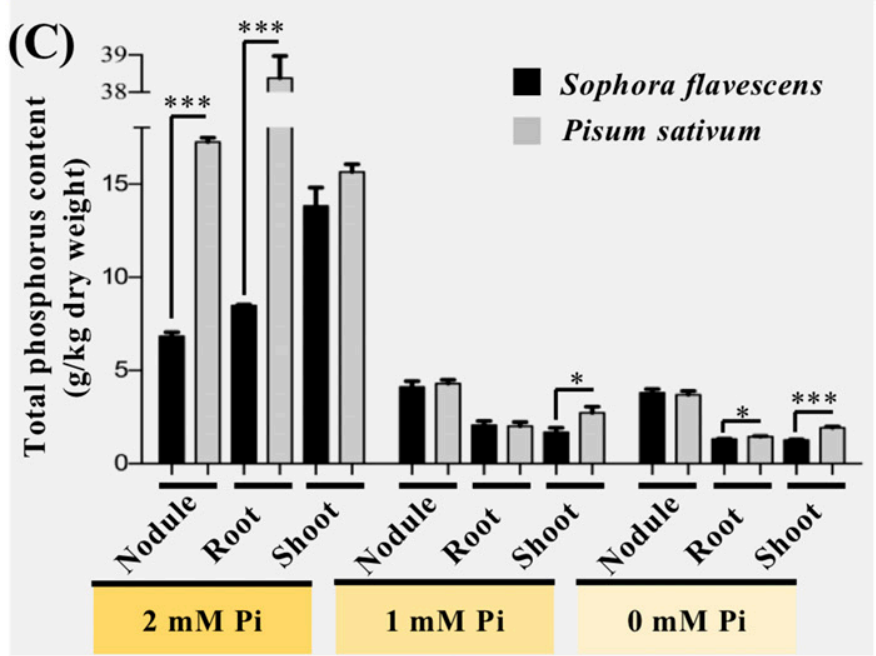

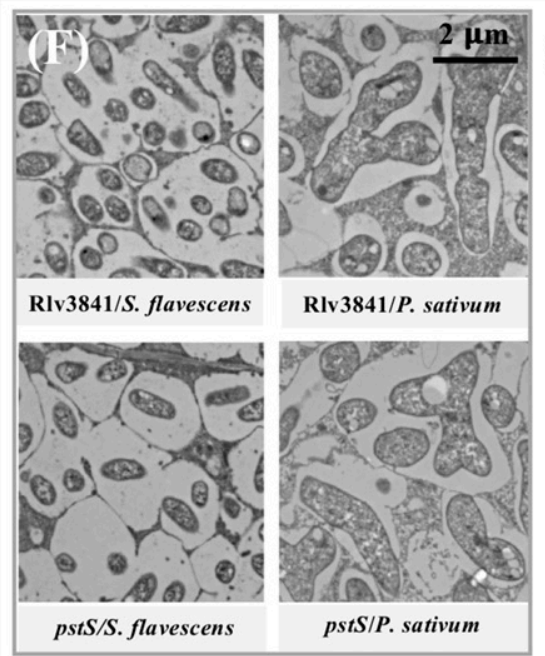

(G)

Number of bacteroids per symbiosome in $S$. flavescens

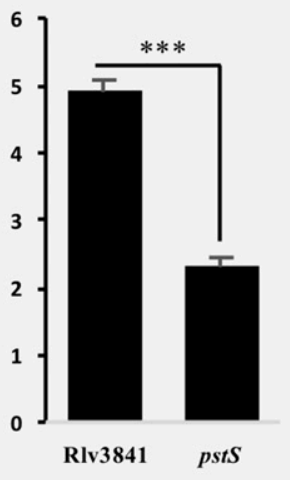

Fig. 6. Rlv3841 bacteroids in nodules of Sophora flavescens and Pisum sativum. A, Longitudinal sections of nodules stained for $\beta$-galactosidase activity Nodules were collected 40 days postinoculation from P. sativum and S. flavescens inoculated with Rlv3841 harboring the promoter fusion pstS::lacZ. B, Quantitative reverse-transcription polymerase chain reaction analysis of transcriptional changes of pstS, phoC, and pit genes of the wild-type Rlv3841 in the minimum medium with limiting inorganic phosphate $(\mathrm{Pi})(0.1 \mathrm{mM} \mathrm{Pi})$ compared with the culture with excess Pi supply $(2 \mathrm{mM} \mathrm{Pi})$. The means of Log2R were based on results of three cultures. C, Total phosphorus content of nodules, roots, and shoots from $S$. flavescens and $P$. sativum plants grown in the vermiculite containing 2, 1 , and $0 \mathrm{mM} \mathrm{KH}_{2} \mathrm{PO}_{4}$. Means were obtained from three to six independent experiments. D, Size of bacteroids and free-living cells of Rlv3841 (100 bacteroids or free-living cells were scored). E, Million CFU per nodule (three nodules were used in each experiment, and the mean of three independent experiments is shown). F, Transmission electron microscopy pictures of 80-nm thin section of nodules (40 days postinoculation) formed by the wild-type Rlv3841 and the $p s t S$ mutant. Scale bar indicates $2 \mu \mathrm{m}$. G, Number of bacteroids per symbiosome in $S$. flavescens nodules ( 84 symbiosomes were scored for each treatment). Error bars represent standard error of mean, and significant difference is indicated ( $t$ test, * and *** indicate $P<0.05$ and 0.001 , respectively). 
collected under $1 \mathrm{mM}$ Pi conditions suggested that the $P$. sativum nodule has a relatively lower Pi concentration in its proximal infected cells than other infected cells (Fig. 6A). Moreover, it has been shown that phosphorus in G. max nodules was higher in the infected region than in the cortical region but most heavily accumulated in the closely associated root cortex cells (Mizukoshi et al. 1995; Ohyama 2017). Therefore, the level of phosphorus content can be heterogeneous in the well-organized nodule structure.

In the model system of Sinorhizobium meliloti-Medicago sativa, the low-affinity Pi transporter Pit was highly expressed in nodules formed by the wild-type $S$. meliloti 2011 or its $p h o B$ mutant, whereas very little expression of PstSCAB and PhoCDET was detected (Yuan et al. 2006). By contrast, within G. max nodules induced by SF45436, high expression levels of Pst and Pho but limited Pit expression were observed. Reverse genetics suggested that any one of three Pi transporters was sufficient for effective symbiosis of $S$. meliloti with Medicago sativa (Yuan et al. 2006), while the pst mutant rather than the pit or phoC mutant of SF45436 was symbiotically defective on G. max. That is to say, three Pi transporters of rhizobia were complementary within Medicago nodules but not within G. $\max$ nodules. Therefore, it is reasonable to conclude that $S$. meliloti bacteroids are not Pi-limited in Medicago nodules (Yuan et al. 2006) but a subsequent general proposal that root nodule bacteria are not Pi limited and the Pit is the main system in planta (Udvardi and Poole 2013) should be updated.

Despite the differences between $S$. meliloti and $S$. fredii in the expression profiles of $\mathrm{Pi}$ transporters in nodules, the PhoBdependent activation of Pst or Pho expression and repression of Pit is conserved in these two species, as demonstrated in earlier studies of S. meliloti (Bardin and Finan 1998; Bardin et al. 1998; Voegele et al. 1997; Yuan et al. 2006) and herein for $S$. fredii. Moreover, derepressing Pit by the mutation of $p h o B$ can be hypothesized to restore the symbiotic defects (shoot dry weight) of the pst-phoC mutant of $S$. meliloti (Bardin and Finan 1998; Yuan et al. 2006) and the pst or pst-phoC mutants of $S$. fredii. However, the growth defects under the limiting $\mathrm{Pi}$ condition and the reduced number of bacteroids per symbiosome for the pst mutant of $S$. fredii could not be fully recovered in the phoB-pst double mutant. This is in line with the evidence that Pst is a high-affinity, high-velocity Pi transporter, compared with the nonspecific Pho and Pit systems (Voegele et al. 1997; Yuan et al. 2006). Although Pst is expressed lower than Pit to avoid toxic levels of Pst-mediated Pi accumulation under excess Pi conditions (diCenzo et al. 2017), accumulating data show that Pst rather than Pit serves as the primary Pi transporter when Pi is in excess (Hsieh and Wanner 2010; McCleary 2017). This view is further supported by a similar growth rate of pst and pst-pit mutants of SF45436 in the medium supplied with $2.0 \mathrm{mM}$ Pi (Fig. 4B). The slower growth rate of the pst-phoC mutant than the pst mutant indicated that PhoCDET also contributes to the adaptation to excess Pi conditions. It should be noted that loss-of-function mutations in the pst genes can lead to the constitutive phosphorylation of $\mathrm{PhoB}$ and subsequent induction of Pho regulon, including phoCDET and repression of pit (Hsieh and Wanner 2010; Yuan et al. 2006). However, pit can be deregulated in the phoB-pst mutant, which exhibited a similar growth rate compared with the wild-type SF45436 under the Pi excess condition.

Contrasting expression patterns of the high-affinity $\mathrm{Pi}$ transporter Pst in determinate nodules of G. max and indeterminate nodules of Medicago sativa inspired us to further quantify pst transcription levels in nitrogen-fixing bacteroids within indeterminate nodules of $C$. cajan, Sophora flavescens, and $P$. sativum, or within determinate nodules of Phaseolus vulgaris, V. unguiculata, and A. hypogaea, despite potential variation in their nodulation dynamics. The transcription profiles of pst were independent of whether the nodule is determinate or indeterminate, and correlated well with differentiation types of bacteroids (either terminally or nonterminally differentiated) reported earlier for these legumes (Oono et al. 2010). These transcriptional patterns of pst are supported by transcriptomic data collected for bacteroids within nodules of G. max (Jiao et al. 2016; Pessi et al. 2007), P. vulgaris (Vercruysse et al. 2011), V. unguiculata ( $\mathrm{Li}$ et al. 2013), and Leucaena leucocephala (Li et al. 2013). In these transcriptomes of nonterminally differentiated bacteroids, the high-affinity $\mathrm{Pi}$ transporter Pst rather than Pit was significantly upregulated in bacteroids compared with free-living cultures. Notably, L. leucocephala nodules are indeterminate ones harboring a consistent meristem similar to those of Medicago sativa (Li et al. 2013), in contrast to those determinate nodules with a transient meristem. Different transcriptional profiles of pst in bacteroids with either terminal or nonterminal differentiations (Fig. 5) are also supported by expression patterns of pstS::lacZ reporters in nodules of G. max (Fig. 3), S. flavescens (Fig. 6), and Pisum sativum (Fig. 6) or a pstS::gusA reporter in Medicago nodules (Yuan et al. 2006). These findings constitute an indication of the correlation between Pi homeostasis and differentiation of bacteroids.

Terminally differentiated bacteroids, characterized by an irreversible loss of cell division ability, genome amplification, and enlarged cell size, are modulated by host-derived NCR peptides such as those found in the model system Sinorhizobium meliloti-Medicago spp. (Mergaert et al. 2006; Van de Velde et al. 2010; Wang et al. 2010). Notably, it has been reported that many prokaryotes, including Shewanella and Vibrio spp., can use extracellular DNA as the sole phosphate source (McDonough et al. 2016; Pinchuk et al. 2008). The ploidy level of Haloferax volcanii and Synechocystis sp. PCC 6803 was elevated at higher phosphate concentrations while a rapid reduction in genome copy number was observed during the growth under limited phosphate conditions (Zerulla et al. 2014, 2016). These findings could raise intriguing hypotheses regarding the contribution of phosphate homeostasis to the ploidy level of bacteroids in legumes such as Medicago sativa and $P$. sativum, though nodule environment is much more complex than free-living cultures. On the other hand, host legumes such as G. max and Sophora flavescens may restrict phosphate supply to avoid overproliferation of bacteroids without terminal differentiation in nodules.

Host-dependent morphologies of bacteroids such as swollen, elongated, spherical, and elongated-branched correlated well with the number and type of symbiotic NCR peptides (Czernic et al. 2015; Montiel et al. 2017). Moreover, CFU of rhizobial populations isolated from nodules have been demonstrated to be an effective indicator for the relative reproductive ability of bacteroids with either terminal or nonterminal differentiations (Montiel et al. 2016). However, NCR-induced differentiation and survival of rhizobia are strongly affected by rhizobial surface polysaccharides (Montiel et al. 2017), the structures of which can be different among diverse rhizobial species (Downie 2010). To avoid the influence of differences in rhizobial strains, Rlv3841 bacteroids within nodules of $S$. flavescens and $P$. sativum were compared in this study. Reproductive ability was significantly higher for Rlv3841 bacteroids from nodules of $S$. flavescens than those from $P$. sativum. This is in line with the evidence that Rlv3841 bacteroids have complete metabolic dependence on $P$. sativum and behave like organelles (Prell et al. 2009). The stronger induction of high-affinity Pi transporter Pst in Rlv3841 bacteroids in $S$. flavescens than in P. sativum may represent one of the largely unexplored adaptive routes, which are crucial for the persistence of bacteroids without terminal differentiation in nodules, as experimentally demonstrated using 
the pstS mutant of Rlv3841 and the pst mutant of Sinorhizobium fredii associated with G. max. Notably, the impaired adaptation of the pstS mutant in Sophora flavescens nodules didn't lead to a significant decrease of symbiotic efficiency regarding shoot dry weight of inoculated plants under test conditions. Although the underlying mechanisms remain unexplored, it has been demonstrated that the Pho regulon involved in phosphate starvation adaptation is upregulated in the pst mutant of diverse bacteria (Hsieh and Wanner 2010; Yuan et al. 2006). This regulon includes genes encoding transporters for secondary phosphorus sources such as phosphonates (phoCDET) and organophosphates (ugpBAECQ). There could be some variation in the pool of secondary phosphorus sources in nodules of different legumes where phosphate-starved bacteroids without terminal differentiation may perform differentially in symbiotic efficiency.

\section{MATERIALS AND METHODS}

\section{Bacterial strains, plasmids, and growth conditions.}

E. coli strains, rhizobia, and plasmids used in this study are listed in Supplementary Table S1. Tryptone-yeast (TY) medium (tryptone at $5 \mathrm{~g} \mathrm{liter}^{-1}$, yeast extract at $3 \mathrm{~g} \mathrm{liter}^{-1}$, and $\mathrm{CaCl}_{2}$ at $0.6 \mathrm{~g} \mathrm{l}^{-1}$ ), or minimum medium (MOPS at $10 \mathrm{~g} / \mathrm{liter}$, mannitol at $10 \mathrm{~g} /$ liter, sodium glutamate at $3.55 \mathrm{~g} /$ liter, $1 \mathrm{mM}$ $\mathrm{MgSO}_{4}, 250 \mu \mathrm{M} \mathrm{CaCl} 2, \mathrm{FeCl}_{3}$ at $10 \mathrm{mg} / \mathrm{liter}, \mathrm{H}_{3} \mathrm{BO}_{3}$ at $3 \mathrm{mg} / \mathrm{liter}, \mathrm{MnSO}_{4} \cdot 4 \mathrm{H}_{2} \mathrm{O}$ at $2.23 \mathrm{mg} / \mathrm{liter}$, biotin at $1 \mathrm{mg} / \mathrm{liter}$, $\mathrm{ZnSO}_{4} .7 \mathrm{H}_{2} \mathrm{O}$ at $0.3 \mathrm{mg} / \mathrm{liter}, \mathrm{NaMoO}_{4} .2 \mathrm{H}_{2} \mathrm{O}$ at $0.12 \mathrm{mg} / \mathrm{liter}$, and $\mathrm{CoCl}_{2} \cdot 6 \mathrm{H}_{2} \mathrm{O}$ at $0.065 \mathrm{mg} / \mathrm{liter} ; \mathrm{pH}$ 7.2) (Krol and Becker 2004) containing $0.1 \mathrm{mM} \mathrm{Pi}$ or $2 \mathrm{mM}$ Pi was used to grow rhizobial strains at $28^{\circ} \mathrm{C}$. Luria-Bertani medium was used to grow E. coli strains at $37^{\circ} \mathrm{C}$. The concentrations of antibiotics used were $30 \mu \mathrm{g} / \mathrm{ml}$ for nalidixic acid, $10 \mu \mathrm{g} / \mathrm{ml}$ for trimethoprim, $50 \mu \mathrm{g} / \mathrm{ml}$ for kanamycin, $30 \mu \mathrm{g} / \mathrm{ml}$ for gentamycin $(\mathrm{Gm})$, and $10 \mu \mathrm{g} / \mathrm{ml}$ for tetracycline.

\section{Construction of the deletion mutants and the lac $Z$ reporters.}

The primers used in this study are shown in Supplementary Table S2. The plasmid pRK2013 (Ditta et al. 1980) was used as the helper plasmid in all conjugation experiments. All cloned fragments harbored by engineered plasmids described below were verified by Sanger sequencing, and the resultant rhizobial mutants were confirmed by PCR.

The SF45436 mutants $\triangle p s t S C A B:: G m$ and $\triangle p h o B:: G m$ had been constructed previously using the cre-lox system (Jiao et al. 2016; Marx and Lidstrom 2002). To obtain the phoB-pst double mutant, the $\mathrm{Gm}$ resistance gene flanked by two loxP sites in the $\triangle p h o B:: G m$ mutant was removed by introducing the plasmid pCM157 expressing Cre recombinase (Marx and Lidstrom 2002). Briefly, pCM157 was introduced into the phoB mutant by conjugation using pRK2013 as the helper plasmid. Transconjugants sensitive to $\mathrm{Gm}$ were further screened for pCM157cured strains, which are tetracycline sensitive. The pstSCAB operon was deleted in this $\triangle p h o B$ mutant by introducing a pCM351 derivative containing PCR fragments encompassing the upstream $\mathrm{N}$-terminal coding region and the downstream $\mathrm{C}$ terminal coding region of the pstSCAB operon amplified using pstUL-pstUR and pstDL-pstDR primers. To construct the phoB-phoC double mutant, the phoC coding region in the $\triangle p h o B$ mutant was inactivated by site-specific insertion mediated by a pVO155 (Oke and Long 1999) derivative harboring a phoC internal fragment amplified using phoCF-phoCR primers. To obtain the phoB-phoC-pst mutant, the pstSCAB operon in this $p h o B-p h o C$ mutant was further deleted using the pCM351 derivative, as described above. To construct the pst-pit mutant, the pit gene in the $\triangle p s t S C A B:: G m$ mutant was inserted by a pVO155 derivative containing pit internal fragment amplified using primers pitF and pitR. The pst-phoC mutant was obtained by introducing the abovementioned pVO155 derivative harboring the $p h o C$ internal fragment into the $\triangle p s t S C A B:: G m$ mutant. The single mutants $p h o C$ and pit were constructed by mobilizing the same pVO155 derivatives described above into the wild-type SF45436.

To construct the $p s t S$ deletion mutant of Rlv3841, a seamless cloning strategy was used to engineer a pJQ200SK (Quandt and Hynes 1993) derivative containing the PCR fragments encompassing the upstream N-terminal coding region and the downstream C-terminal coding region of pstS amplified using primers 3841 pstUF-UR and 3841pstDF-DR. The primers 3841 pstUF and 3841pstDR contained 5' sequences corresponding to two ends of pJQ200SK produced by SmaI digestion, and the primers 3841pstUR and 3841pstDF had reversely complemented $5^{\prime}$ sequences of $20 \mathrm{bp}$ in length. PCR fragments amplified using these two sets of primers and the SmaI-digested pJQ200SK were subject to seamless assembly using Master Assembly Mix (Clone Smarter) at $50^{\circ} \mathrm{C}$ for 15 min. Cooled products were transformed into $E$. coli, then mobilized into the wild-type Rlv3841 by conjugation. Gmresistant transconjugants with a single crossover were subject to further sucrose counterselection for double recombinants using $5 \%$ sucrose.

To construct $l a c Z$ reporters with promoter regions of $p s t S$, phoC, and pit from SF45436, or that of pstS from Rlv3841, the in-frame lacZ translational fusion plasmid pGD926 (Ditta et al. 1985) was used. PCR fragments amplified using primers GD45436pstSF-R, GD45436phoCF-R, GD45436pitF-R, or GD3841pstSF-R were individually assembled with the BamHIHindIII-digested pGD926 through the seamless assembly procedure described above. The resultant derivatives of pGD926 were mobilized into wild-type SF45436, the phoB mutant, or wild-type Rlv3841 by conjugation.

\section{Plant assays, CFU from nodules,} and electronic microscopy.

Seed of legumes were surface sterilized in $3 \%$ ( $\mathrm{vol} / \mathrm{vol})$ $\mathrm{NaClO}$ solution, allowed to germinate, and inoculated with $1 \mathrm{ml}$ of culture of the indicated rhizobial strain at an optical density at $600 \mathrm{~nm}\left(\mathrm{OD}_{600}\right)=0.2$. Plants were grown in vermiculite moistened with low-N nutrient solution in Leonard jars at $24^{\circ} \mathrm{C}$ with 12 -h day and night cycles (Vincent 1970). At 30 dpi, plant shoots were dried at $65^{\circ} \mathrm{C}$ for 5 days; then, dry weight was determined. The number of rhizobial cells in nodules being able to form colonies was determined by plating the rhizobial population from 40 dpi nodules of S. flavescens and P. sativum. Three nodules were used in each experiment, and three independent experiments were performed.

Ultrathin sections of root nodules collected at 30 or $40 \mathrm{dpi}$ as indicated were prepared using a Leica Ultracut C6i and observed in a JEM-1230 transmission electron microscope (TEM), as described earlier ( $\mathrm{Li}$ et al. 2013). The size of Rlv3841 bacteroids in $P$. sativum and S. flavescens were scored from 100 bacteroids. To determine the number of Rlv3841 or pstS bacteroids per symbiosome in $S$. flavescens nodules, 84 symbiosomes from seven TEM views were scored. Similarly, the number of Sinorhizobium fredii bacteroids per symbiosome in G. max nodules was scored from 100 symbiosomes.

\section{Expression of $l a c Z$ reporters in nodules.}

Plants of G. max, P. sativum, and Sophora flavescens were inoculated with corresponding rhizobial strains carrying the pGD926 derivatives, which express pstS::lacZ, phoC::lacZ, or pit::lacZ reporter gene fusion. Nodules were collected at 30 or $40 \mathrm{dpi}$ as indicated, and fixed with $2 \%$ (vol/vol) glutaraldehyde in Z' buffer (0.1 M potassium phosphate buffer, $\mathrm{pH} 7.4 ; 1 \mathrm{mM}$ 
$\mathrm{MgSO}_{4}$; and $10 \mathrm{mM} \mathrm{KCl}$ ). Then, 80- $\mu \mathrm{m}$-thick longitudinal nodule sections were prepared using a vibrating-blade microtome (VT1000S; Leica), stained for $1 \mathrm{~h}$ at $28^{\circ} \mathrm{C}$, and observed under a light microscope.

\section{qRT-PCR.}

For bacteroid samples, nodules collected at 30 dpi were subject to RNA extraction using the QIAGEN RNeasy mini kit. For free-living cultures, rhizobial cells were first cultured in TY medium, then inoculated, at a starting density of $\mathrm{OD}_{600}=0.05$, into minimal medium containing either 0.1 or $2 \mathrm{mM}$ Pi. Cultures were collected at midlog phase $\left(\mathrm{OD}_{600}=\right.$ approximately 0.6 to 0.7 ), and RNA was then extracted using the QIAGEN RNeasy mini kit according to the manufacturer's instructions. RNA quality was assessed by using Nanophotometer Pearl. Single-strand cDNA was synthesized by using the StarScript II First-Strand cDNA Synthesis Mix (Genstar). qPCR was performed by using $2 \times$ RealStar Green Mixture (Genstar) and LightCycler 480 II. The mean of crossing point values for the control gene 16S ribosomal RNA was used as the reference for normalization of gene expression, as described earlier ( $\mathrm{Li}$ et al. 2013). Three independent biological replicates were analyzed in all qRT-PCR experiments.

\section{Phosphorus content determination.}

Seed of $P$. sativum and $S$. flavescens were surface sterilized as mentioned above, then inoculated with Rlv3841. Plants were grown in vermiculate moistened with low-N nutrient solution containing 2, 1, or $0 \mathrm{mM} \mathrm{KH_{2 }} \mathrm{PO}_{4}$. After 40 dpi, plant nodules, roots, and shoots were collected and dried at $65^{\circ} \mathrm{C}$ for 5 days. These dry samples were ground into powder, 0.2 to $0.3 \mathrm{~g}$ of which was then weighed and placed in a digestion vessel of the CEM Microwave Accelerated Reaction System (CEM-MARS-6; CEM Corporation) with $8 \mathrm{ml}$ of concentrated nitric acid. Overnight samples were digested at $160^{\circ} \mathrm{C}$ till the volume reached $2 \mathrm{ml}$. The cooled samples were diluted with water to $50 \mathrm{ml}$, the phosphorus content of which was analyzed using a ThermoiCAP6300 inductively coupled plasma radial spectrophotometer (Thermo Fisher Scientific) at National Energy R\&D Center for Biomass, China Agricultural University, Beijing, China. Three to six replicated biological samples were determined.

\section{ACKNOWLEDGMENTS}

We thank J. P. W. Young and C. Staehelin for providing rhizobial strains Rlv3841 and NGR234 and M. Zhang and C. Yang for providing seed of JD17.

\section{LITERATURE CITED}

Alunni, B., and Gourion, B. 2016. Terminal bacteroid differentiation in the legume-rhizobium symbiosis: nodule-specific cysteine-rich peptides and beyond. New Phytol. 211:411-417.

Bardin, S., Dan, S., Osteras, M., and Finan, T. M. 1996. A phosphate transport system is required for symbiotic nitrogen fixation by Rhizobium meliloti. J. Bacteriol. 178:4540-4547.

Bardin, S. D., and Finan, T. M. 1998. Regulation of phosphate assimilation in Rhizobium (Sinorhizobium) meliloti. Genetics 148:1689-1700.

Bardin, S. D., Voegele, R. T., and Finan, T. M. 1998. Phosphate assimilation in Rhizobium (Sinorhizobium) meliloti: Identification of a pit-like gene. J. Bacteriol. 180:4219-4226.

Crespo-Rivas, J. C., Guefrachi, I., Mok, K. C., Villaécija-Aguilar, J. A., Acosta-Jurado, S., Pierre, O., Ruiz-Sainz, J. E., Taga, M. E., Mergaert, P., and Vinardell, J. M. 2016. Sinorhizobium fredii HH103 bacteroids are not terminally differentiated and show altered $\mathrm{O}$-antigen in nodules of the inverted repeat-lacking clade legume Glycyrrhiza uralensis. Environ. Microbiol. 18:2392-2404.

Czernic, P., Gully, D., Cartieaux, F., Moulin, L., Guefrachi, I., Patrel, D., Pierre, O., Fardoux, J., Chaintreuil, C., Nguyen, P., Gressent, F., Da Silva, C., Poulain, J., Wincker, P., Rofidal, V., Hem, S., Barrière, Q.,
Arrighi, J. F., Mergaert, P., and Giraud, E. 2015. Convergent evolution of endosymbiont differentiation in Dalbergioid and inverted repeat-lacking clade legumes mediated by nodule-specific cysteine-rich peptides. Plant Physiol. 169:1254-1265.

diCenzo, G. C., Sharthiya, H., Nanda, A., Zamani, M., and Finan, T. M 2017. PhoU allows rapid adaptation to high phosphate concentrations by modulating PstSCAB transport rate in Sinorhizobium meliloti. J. Bacteriol. 199:e00143-e17.

Ditta, G., Schmidhauser, T., Yakobson, E., Lu, P., Liang, X. W., Finlay, D. R., Guiney, D., and Helinski, D. R. 1985. Plasmids related to the broad host range vector, pRK290, useful for gene cloning and for monitoring gene expression. Plasmid 13:149-153.

Ditta, G., Stanfield, S., Corbin, D., and Helinski, D. R. 1980. Broad host range DNA cloning system for gram-negative bacteria: Construction of a gene bank of Rhizobium meliloti. Proc. Natl. Acad. Sci. U.S.A. 77: 7347-7351.

Downie, J. A. 2010. The roles of extracellular proteins, polysaccharides and signals in the interactions of rhizobia with legume roots. FEMS Microbiol. Rev. 34:150-170.

Galibert, F., Finan, T. M., Long, S. R., Puhler, A., Abola, P., Ampe, F., Barloy-Hubler, F., Barnett, M. J., Becker, A., Boistard, P., Bothe, G., Boutry, M., Bowser, L., Buhrmester, J., Cadieu, E., Capela, D., Chain, P., Cowie, A., Davis, R. W., Dreano, S., Federspiel, N. A., Fisher, R. F., Gloux, S., Godrie, T., Goffeau, A., Golding, B., Gouzy, J., Gurjal, M., Hernandez-Lucas, I., Hong, A., Huizar, L., Hyman, R. W., Jones, T., Kahn, D., Kahn, M. L., Kalman, S., Keating, D. H., Kiss, E., Komp, C., Lelaure, V., Masuy, D., Palm, C., Peck, M. C., Pohl, T. M., Portetelle, D., Purnelle, B., Ramsperger, U., Surzycki, R., Thebault, P., Vandenbol, M., Vorholter, F. J., Weidner, S., Wells, D. H., Wong, K., Yeh, K. C., and Batut, J. 2001. The composite genome of the legume symbiont Sinorhizobium meliloti. Science 293:668-672.

Haag, A. F., Arnold, M. F. F., Myka, K. K., Kerscher, B., Dall'Angelo, S., Zanda, M., Mergaert, P., and Ferguson, G. P. 2013. Molecular insights into bacteroid development during Rhizobium-legume symbiosis. FEMS Microbiol. Rev. 37:364-383.

Hsieh, Y.-J., and Wanner, B. L. 2010. Global regulation by the sevencomponent Pi signaling system. Curr. Opin. Microbiol. 13:198-203.

Jiao, J., Wu, L. J., Zhang, B., Hu, Y., Li, Y., Zhang, X. X., Guo, H. J., Liu, L. X., Chen, W. X., Zhang, Z., and Tian, C. F. 2016. MucR is required for transcriptional activation of conserved ion transporters to support nitrogen fixation of Sinorhizobium fredii in soybean nodules. Mol. Plant-Microbe Interact. 29:352-361.

Jiao, Y. S., Liu, Y. H., Yan, H., Wang, E. T., Tian, C. F., Chen, W. X., Guo, B. L., and Chen, W. F. 2015. Rhizobial diversity and nodulation characteristics of the extremely promiscuous legume Sophora flavescens. Mol. Plant-Microbe Interact. 28:1338-1352.

Kouas, S., Labidi, N., Debez, A., Abdelly, C., Kouas, S., and Labidi, N. 2005. Effect of $P$ on nodule formation and $N$ fixation in bean. Agron. Sustain. Dev. 25:389-393.

Krol, E., and Becker, A. 2004. Global transcriptional analysis of the phosphate starvation response in Sinorhizobium meliloti strains 1021 and 2011. Mol. Genet. Genomics 272:1-17.

Lamarche, M. G., Wanner, B. L., Crépin, S., and Harel, J. 2008. The phosphate regulon and bacterial virulence: A regulatory network connecting phosphate homeostasis and pathogenesis. FEMS Microbiol Rev. 32:461-473.

Li, Y., Tian, C. F., Chen, W. F., Wang, L., Sui, X. H., and Chen, W. X. 2013. High-resolution transcriptomic analyses of Sinorhizobium sp. NGR234 bacteroids in determinate nodules of Vigna unguiculata and indeterminate nodules of Leucaena leucocephala. PLoS One 8:e70531.

López-Arredondo, D. L., Leyva-González, M. A., González-Morales, S. I., López-Bucio, J., and Herrera-Estrella, L. 2014. Phosphate nutrition: Improving low-phosphate tolerance in crops. Annu. Rev. Plant Biol. 65:95-123.

Martínez-Romero, E., Segovia, L., Mercante, F. M., Franco, A. A., Graham, P., and Pardo, M. a 1991. Rhizobium tropici, a novel species nodulating Phaseolus vulgaris L. beans and Leucaena sp. trees. Int. J. Syst. Bacteriol. 41:417-426.

Marx, C. J., and Lidstrom, M. E. 2002. Broad-host-range cre-lox system for antibiotic marker recycling in gram-negative bacteria. Biotechniques 33: 1062-1067

McCleary, W. R. 2017. Molecular mechanisms of phosphate homeostasis in Escherichia coli. Pages 333-357 in: Escherichia coli-Recent Advances on Physiology, Pathogenesis and Biotechnological Applications. A. Samie, ed. InTechOpen.

McDonough, E., Kamp, H., and Camilli, A. 2016. Vibrio cholerae phosphatases required for the utilization of nucleotides and extracellular DNA as phosphate sources. Mol. Microbiol. 99:453-469. 
Mergaert, P., Uchiumi, T., Alunni, B., Evanno, G., Cheron, A., Catrice, O., Mausset, A. E., Barloy-Hubler, F., Galibert, F., Kondorosi, A., and Kondorosi, E. 2006. Eukaryotic control on bacterial cell cycle and differentiation in the Rhizobium-legume symbiosis. Proc. Natl. Acad. Sci. U.S.A. 103:5230-5235.

Mizukoshi, K., Nishiwaki, T., Ohtake, N., Minagawa, R., Ikarashi, T., Ohyama, T., and Ohyama, T. 1995. Nitrate transport pathway into soybean nodules traced by tungstate and ${ }^{15} \mathrm{NO}_{3}^{-}$. Soil Sci. Plant Nutr. 41 : 75-88.

Montiel, J., Downie, J. A., Farkas, A., Bihari, P., Herczeg, R., Bálint, B., Mergaert, P., Kereszt, A., and Kondorosi, É. 2017. Morphotype of bacteroids in different legumes correlates with the number and type of symbiotic NCR peptides. Proc. Natl. Acad. Sci. U.S.A. 114:5041-5046.

Montiel, J., Szücs, A., Boboescu, I. Z., Gherman, V. D., Kondorosi, É., and Kereszt, A. 2016. Terminal bacteroid differentiation is associated with variable morphological changes in legume species belonging to the inverted repeat-lacking clade. Mol. Plant-Microbe Interact. 29:210-219.

Ohyama, T. 2017. The role of legume-Rhizobium symbiosis in sustainable agriculture. Pages 1-20 in: Legume Nitrogen Fixation in Soils with Low Phosphorus Availability. S. Sulieman and L. S. Tran, eds. Springer Nature, Cham, Switzerland.

Oke, V., and Long, S. R. 1999. Bacterial genes induced within the nodule during the Rhizobium-legume symbiosis. Mol. Microbiol. 32:837-849.

Oono, R., Schmitt, I., Sprent, J. I., and Denison, R. F. 2010. Multiple evolutionary origins of legume traits leading to extreme rhizobial differentiation. New Phytol. 187:508-520.

Pessi, G., Ahrens, C. H., Rehrauer, H., Lindemann, A., Hauser, F., Fischer, H. M., and Hennecke, H. 2007. Genome-wide transcript analysis of Bradyrhizobium japonicum bacteroids in soybean root nodules. Mol. Plant-Microbe Interact. 20:1353-1363.

Pinchuk, G. E., Ammons, C., Culley, D. E., Li, S. M., McLean, J. S., Romine, M. F., Nealson, K. H., Fredrickson, J. K., and Beliaev, A. S. 2008. Utilization of DNA as a sole source of phosphorus, carbon, and energy by Shewanella spp.: Ecological and physiological implications for dissimilatory metal reduction. Appl. Environ. Microbiol. 74: 1198-1208.

Prell, J., White, J. P., Bourdes, A., Bunnewell, S., Bongaerts, R. J., and Poole, P. S. 2009. Legumes regulate Rhizobium bacteroid development and persistence by the supply of branched-chain amino acids. Proc. Natl. Acad. Sci. U.S.A. 106:12477-12482.

Pueppke, S. G., and Broughton, W. J. 1999. Rhizobium sp. strain NGR234 and $R$. fredii USDA257 share exceptionally broad, nested host ranges. Mol. Plant-Microbe Interact. 12:293-318.

Qi, W., Baldwin, S. A., Muench, S. P., and Baker, A. 2016. Pi sensing and signalling: From prokaryotic to eukaryotic cells. Biochem. Soc. Trans. 44:766-773.

Quandt, J., and Hynes, M. F. 1993. Versatile suicide vectors which allow direct selection for gene replacement in gram-negative bacteria. Gene 127:15-21.

Remigi, P., Zhu, J., Young, J. P. W., and Masson-Boivin, C. 2016. Symbiosis within symbiosis: Evolving nitrogen-fixing legume symbionts. Trends Microbiol. 24:63-75.

Sa, T. M., and Israel, D. W. 1991. Energy status and functioning of phosphorus-deficient soybean nodules. Plant Physiol. 97:928-935.
Santos-Beneit, F. 2015. The Pho regulon: A huge regulatory network in bacteria. Front. Microbiol. 6:402.

Sulieman, S., Schulze, J., and Tran, L. S. P. 2013. Comparative analysis of the symbiotic efficiency of Medicago truncatula and Medicago sativa under phosphorus deficiency. Int. J. Mol. Sci. 14:5198-5213.

Sulieman, S., and Tran, L. S. P. 2015. Phosphorus homeostasis in legume nodules as an adaptive strategy to phosphorus deficiency. Plant Sci. 239: 36-43.

Taylor, P., Chekabab, S. M., Harel, J., Dozois, C. M., Chekabab, S. M., Harel, J., and Dozois, C. M. 2014. Interplay between genetic regulation of phosphate homeostasis and bacterial virulence. Virulence 5:786-793.

Udvardi, M., and Poole, P. S. 2013. Transport and metabolism in legumerhizobia symbioses. Annu. Rev. Plant Biol. 64:781-805.

Van de Velde, W., Zehirov, G., Szatmari, A., Debreczeny, M., Ishihara, H., Kevei, Z., Farkas, A., Mikulass, K., Nagy, A., Tiricz, H., SatiatJeunemaître, B., Alunni, B., Bourge, M., Kucho, K., Abe, M., Kereszt, A., Maroti, G., Uchiumi, T., Kondorosi, E., and Mergaert, P. 2010. Plant peptides govern terminal differentiation of bacteria in symbiosis. Science 327:1122-1126.

Vercruysse, M., Fauvart, M., Beullens, S., Braeken, K., Cloots, L., Engelen, K., Marchal, K., and Michiels, J. 2011. A comparative transcriptome analysis of Rhizobium etli bacteroids: Specific gene expression during symbiotic nongrowth. Mol. Plant-Microbe Interact. 24:1553-1561.

Vincent, J. M. 1970. A Manual for the Practical Study of Root Nodule Bacteria. Blackwell, Oxford, UK.

Voegele, R. T., Bardin, S., and Finan, T. M. 1997. Characterization of the Rhizobium (Sinorhizobium) meliloti high- and low-affinity phosphate uptake systems. J. Bacteriol. 179:7226-7232.

Wang, D., Griffitts, J., Starker, C., Fedorova, E., Limpens, E., Ivanov, S., Bisseling, T., and Long, S. 2010. A nodule-specific protein secretory pathway required for nitrogen-fixing symbiosis. Science 327:1126-1129.

Wang, R., Chang, Y. L., Zheng, W. T., Zhang, D., Zhang, X. X., Sui, X. H., Wang, E. T., Hu, J. Q., Zhang, L. Y., and Chen, W. X. 2013. Bradyrhizobium arachidis sp. nov., isolated from effective nodules of Arachis hypogaea grown in China. Syst. Appl. Microbiol. 36:101-105.

Young, J. P. W., Crossman, L. C., Johnston, A. W. B., Thomson, N. R., Ghazoui, Z. F., Hull, K. H., Wexler, M., Curson, A. R., Todd, J. D., Poole, P. S., Mauchline, T. H., East, A. K., Quail, M. A., Churcher, C., Arrowsmith, C., Cherevach, I., Chillingworth, T., Clarke, K., Cronin, A., Davis, P., Fraser, A., Hance, Z., Hauser, H., Jagels, K., Moule, S., Mungall, K., Norbertczak, H., Rabbinowitsch, E., Sanders, M., Simmonds, M., Whitehead, S., and Parkhill, J. 2006. The genome of Rhizobium leguminosarum has recognizable core and accessory components. Genome Biol. 7:R34.

Yuan, Z. C., Zaheer, R., and Finan, T. M. 2006. Regulation and properties of PstSCAB, a high-affinity, high-velocity phosphate transport system of Sinorhizobium meliloti. J. Bacteriol. 188:1089-1102.

Zerulla, K., Chimileski, S., Näther, D., Gophna, U., Papke, R. T., and Soppa, J. 2014. DNA as a phosphate storage polymer and the alternative advantages of polyploidy for growth or survival. PLoS One 9:e94819.

Zerulla, K., Ludt, K., and Soppa, J. 2016. The ploidy level of Synechocystis sp. PCC 6803 is highly variable and is influenced by growth phase and by chemical and physical external parameters. Microbiology 162: 730-739. 